\title{
Trigger Team Activation for Acute Patients. A Cohort Study at a Level 1 University Hospital in Denmark
}

\section{Stefan Posth ( $\sim$ stefan.posth@rsyd.dk)}

University of Southern Denmark https://orcid.org/0000-0002-4325-038X

\section{Lonnie Froberg}

Odense University Hospital: Odense Universitetshospital

\section{Søren Bak}

Odense University Hospital: Odense Universitetshospital

\section{Lisette Okkels Jensen}

Odense University Hospital: Odense Universitetshospital

\section{Mikkel Brabrand}

Odense University Hospital: Odense Universitetshospital

\section{Annmarie Lassen}

Odense University Hospital: Odense Universitetshospital

\section{Research article}

Keywords: trigger team, medical emergency patient, trauma, stroke, STEMI

Posted Date: November 19th, 2020

DOI: https://doi.org/10.21203/rs.3.rs-108214/v1

License: (c) (1) This work is licensed under a Creative Commons Attribution 4.0 International License. Read Full License 


\section{Trigger team activation for acute patients. A cohort study at a}

\section{2 level 1 university hospital in Denmark}

3 Stefan Posth ${ }^{1}$, Lonnie Froberg ${ }^{2}$, Søren Bak ${ }^{3}$, Lisette Okkels Jensen ${ }^{4}$, Mikkel Brabrand ${ }^{5}$, Annmarie Lassen ${ }^{1}$

$4 \quad{ }^{1}$ Clinical Institute, University of Southern Denmark and Department of Emergency Medicine, Odense

5 University Hospital, Denmark; Corresponding author

$6 \quad{ }^{2}$ Department of Orthopaedic Surgery, Odense University Hospital, Denmark

$7 \quad{ }^{3}$ Department of Neurology, Odense University Hospital, Denmark

$8{ }^{4}$ Department of Cardiology, Odense University Hospital, Denmark

$9{ }^{5}$ Institute of Regional Health Research, University of Southern Denmark; Department of Emergency Medicine,

10 Hospital of South West Jutland, Denmark; Department of Emergency Medicine, Odense University Hospital,

11 Denmark

12

13

14

15 Abstract:

16 Background: While patients with major trauma, suspected ST-elevation myocardial infarction (STEMI) or

17 stroke have implemented pathways for quick access to specialized trigger teams, other patients with

18 time dependent diseases present with less obvious aetiology and have less documented pathways at

19 arrival to the hospital. 
20 Aim: To describe the number, diagnosis and prognosis of patients arriving to the trigger teams for

21 trauma, suspected STEMI, suspected stroke, and Medical emergency patients (MEP)

22 Method: Retrospective cohort study of all patients who between November 2012 and September 2015

23 had either a trauma, STEMI, stroke or MEP trigger team activation at arrival to Odense University

24 Hospital - a level 1 trauma centre, a direct referral centre for patients with suspected STEMI to the

25 catheterization laboratory or stroke, and with a trigger team at emergency department arrival of MEP

26 patients from the local area.

27 Results: There were 8,075 activations of a trigger team at hospital arrival, median 7.6 calls per day

28 (range 1-18); 16.7\% trauma, 28.3\% STEMI, 19.7\% stroke and 35.3\% MEP calls. This corresponds to

$29161 / 100,000$ person years (py) with trauma calls, 64/100,000 py STEMI calls, 72/100,000 py stroke calls

30 and $340 / 100,000$ py MEP calls.

31 Patients from the different calls had a 30-day mortality of $12 \%$ (trauma), $8 \%$ (STEMI), $5 \%$ (stroke), and

$32 \quad 25 \%(\mathrm{MEP})$.

33 Whereas patients from trauma, STEMI and stroke calls were mainly discharged within a few ICD10

34 (International classification of diseases, version 10) main coding areas, patients from MEP calls had

35 discharge diagnosis within many different ICD10 main coding areas.

36 Conclusion: Trauma, STEMI and stroke trigger teams are used at a daily base, treat a prehospitally well-

37 defined patient population and have a relatively low 7-day mortality. Patients with MEP calls are more

38 frequent, have a diverse aetiology and a higher mortality than patients in the other trigger teams.

39 Keywords: trigger team, medical emergency patient, trauma, stroke, STEMI

40 


\section{Background:}

44 People outside the hospital in medical distress activate the Emergency Medical System. In Denmark, an

45 ambulance is dispatched, and if the situation is deemed severe enough, a physician-manned unit

46 (Mobile Emergency Care Unit) is deployed as well. However, when the patients arrive at the Emergency

47 Hospital, their paths split.

48 A dedicated trauma team, headed by an orthopaedic surgeon or an anaesthesiologist, receives trauma

49 patients (1). Patients with ST-elevation myocardial infarction (STEMI) are sent to the cardiac

50 catheterization laboratory, and a neurologist promptly evaluates stroke patients for potential

51 thrombolysis.

52 Patients with less differentiated problems, medical emergency patients (MEP), which include many of

53 the patients with shock, respiratory distress or depressed level of consciousness, do not fall into any of

54 these categories. They are received in the emergency department (ED) by emergency physicians.

55 However, they are also in an acute life-threatening situation and require immediate care (2-6). As their

56 path through the ED is not as well defined as for patients with trauma, stroke or STEMI, many EDs have

57 implemented each their dedicated pathway or a dedicated team to receive, assess and treat these

58 patients $(7,8)$. This is especially important as reliably matching patient needs with ED resources in time-

59 dependent illness is a critical component of a coordinated emergency care system (9). In contrast to the

60 implemented pathways for trauma, STEMI and stroke teams, most MEP teams are locally developed and

61 are scarcely described and evaluated in a general setting. The aim of the study is to describe the

62 number, diagnosis and prognosis of patients arriving to the trigger teams for trauma, suspected STEMI,

63 suspected stroke, and MEP. 
67 Methods:

68 This is a population-based cohort study. Odense University Hospital (OUH) is one of three centres in the

69 Region of Southern Denmark performing stroke thrombolysis and the only centre receiving level 1

70 orthopaedic trauma, and patients with prehospitally diagnosed STEMI. MEP can be admitted in five

71 different hospitals in the region.

72 All MEP and patients with trauma, suspected stroke as well as suspected STEMI who arrived to a

73 specialized team response at OUH between 1 November 2012 and 30 September 2015 were included in

74 the study. Patients under the age of 18 were excluded. Data on outcome (mortality) was extracted from

75 Danish Civil Registration System (10) and all hospital contacts from the National Patient Registry (LPR)

76 (11). In Denmark, all inhabitants have access to universal free healthcare and use their personal

77 identification number in relation to the contacts.

78 Composition of the different trigger teams is poorly defined; no international consensus exists, just 79 suggestions for trauma teams (12).

80 The trauma trigger team at OUH consists of an orthopaedic surgeon, anaesthesiologist, anaesthesiology

81 nurse, two ED nurses, radiologist, two radiology technicians, laboratory assistant, orderly and a

82 secretary. Other specialists can be summoned to the resuscitation room as needed. Patients are

83 received in the EDs resuscitation room. The inclusion criteria for the team call are a patient after a

84 physical trauma with an objective or suspected life-threatening injury or an injury of more than one

85 organ system (penetrating trauma head / torso, massive bleeding, massive blunt trauma, instable pelvis

86 fracture, two or more major fractures, flail chest, $A B C D E$ instable, fall $>6 \mathrm{~m}$, trapped $>30$ minutes). 
87 During the inclusion period, patients arrived from the northern part of the island of Funen, Denmark

88 (primary trauma) and from the whole region of Southern Denmark (secondary trauma). There are

89290,000 inhabitants living in the catchment area for primary trauma, and 1,220,000 for secondary

90 trauma (figure 1$)$.

91

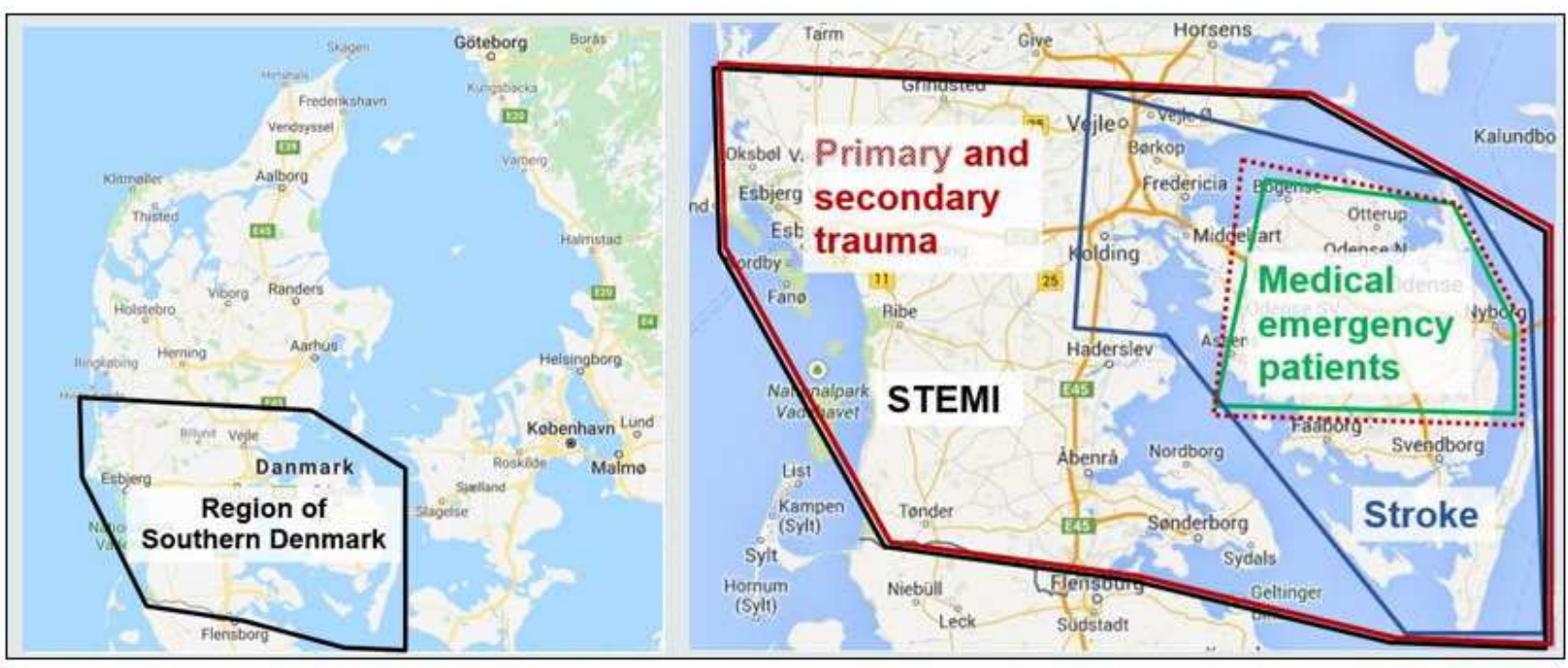

Figure 1: Catchment area

STEMI: ST-elevation myocardial infarction patients

The OUH stroke trigger team consists of a thrombolysis consultant from the neurology department, ED nurse, neurology nurse, laboratory assistant and secretary. Inclusion criteria for the team call is a suspected acute ischaemic stroke, less than 4.5 hours after debut of symptoms, and a previously selfreliant patient. During the inclusion period, patients were received in the EDs computed tomography scanner and resuscitation room. They arrived from the island of Funen, including the outlying smaller islands, and the eastern part of southern Jutland, Denmark. This amounts to a catchment area of 754,000 inhabitants (figure 1). 
102 The STEMI trigger team at OUH consists of an interventional cardiologist and three specialised nurses.

103 The patients are received in the cardiac catheterization laboratory following prehospital visitation. The

104 inclusion criteria for the team call are typical symptoms for myocardial infarction for less than 12 hours

105 and electrocardiogram findings suggesting STEMI. Also, patients with cardiac arrest with a suspected

106 cardiac reason are included in this group. The patients arrive from the whole region of Southern

107 Denmark, with 1,220,000 inhabitants (figure 1).

108 The MEP trigger team at OUH consists of a medical consultant on duty in the ED, senior medical

109 resident, two ED nurses, secretary, orderly, laboratory assistant and a radiology technician. If the

110 patient's airway is threatened, or if the patient is unconscious with a Glasgow Coma scale of less than 8,

111 an anaesthesiologist and an anaesthesiology nurse are part of the MEP trigger team. Patients are

112 received in the EDs resuscitation room. The inclusion criteria for the team call is one or more of the

113 following: threatened airway, systolic blood pressure $<80 \mathrm{mmHg}$, pulse rate $>130 / \mathrm{min}$, respiratory rate

$114>35 / \mathrm{min}$, oxygen saturation $<80 \%$ or Glasgow Coma Scale $<8$. Also, patients with cardiac arrest with a

115 suspected non-cardiogenic reason are included in this group. The patients arrive from the northern part

116 of the island of Funen, Denmark, resulting in a catchment area of 290,000 inhabitants (figure 1).

117 Information on inhabitants in the different catchment areas are retrieved from Statistics Denmark

118 (numbers from 1 January 2018).

119 Data was analyzed using Stata/IC (version 15).

123 Results: 
124 In the 35 observation months, there were 8,075 trigger team activations for adult patients at arrival to

125 OUH, range 1-18, median 7.6 per day. An additional trigger team was activated in 36 cases (0.5\%), for example an initial trauma trigger call was supplemented with a MEP trigger call.

127 Whereas 7,568 patients (94.7\%) experienced one trigger call during inclusion period, 374 (4.6\%)

128 experienced two trigger calls at different points of time, and two patients experienced 13.

130 The demographic details of patients involved in all trigger calls are shown in table $1.16 .7 \%$ of all trigger

131 team activations were trauma, 28.3\% STEMI, 19.7\% stroke and 35.3\% MEP. This corresponds to

$132161 / 100,000$ person years (py) with trauma calls, 64/100,000 py STEMI calls, $72 / 100,000$ py stroke calls

133 and $340 / 100,000$ py MEP calls.

134 Patients from the different calls had a mean age of 48 for trauma calls, and a similar mean age of around

13565 for the other calls. Most of the trauma and STEMI call patients were male, whereas the female / male

136 distribution was more even for stroke and MEP calls. Seven-day mortality was lowest for stroke patients

137 and highest for MEP. Transferral rate for patients to the intensive care unit was lowest for stroke and

138 highest for trauma call patients.

Table 1: Demographic information on all included patients

\begin{tabular}{|c|c|c|c|c|c|c|c|c|c|c|}
\hline $\begin{array}{l}\text { Trigger } \\
\text { team }\end{array}$ & 资 & 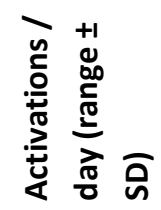 & 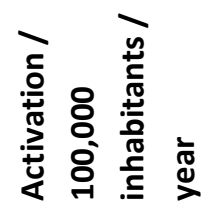 & 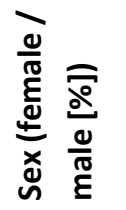 & 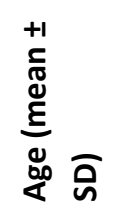 & 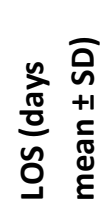 & 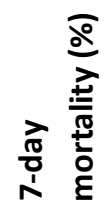 & 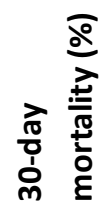 & 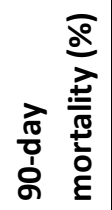 & $\frac{\bar{a}}{\partial}$ \\
\hline Trauma & 1,347 & $\begin{array}{l}1.3(1-6 \pm \\
1.0)\end{array}$ & 161 & $29 / 71$ & $48 \pm 20$ & $\begin{array}{l}10.5 \pm \\
18.6\end{array}$ & 10.4 & 12 & 13.2 & 37.1 \\
\hline
\end{tabular}




\begin{tabular}{|l|l|l|l|l|l|l|l|l|l|l|}
\hline STEMI & 2,284 & $\begin{array}{l}2.2(1-8 \pm \\
1.4)\end{array}$ & 64 & $27 / 73$ & $65 \pm 13$ & $\begin{array}{l}5.2 \pm \\
7.2\end{array}$ & 5.7 & 7.7 & 9.1 & 10.9 \\
\hline Stroke & 1,594 & $\begin{array}{l}1.5(1-7 \pm \\
1.1)\end{array}$ & 72 & $42 / 58$ & $65 \pm 16$ & $12 \pm 22$ & 3.4 & 5.3 & 6.8 & 7.9 \\
\hline MEP & 2,850 & $\begin{array}{l}2.7(1-9 \pm \\
1.5)\end{array}$ & 340 & $47 / 53$ & $66 \pm 18$ & $\begin{array}{l}7.4 \pm \\
11\end{array}$ & 16.9 & 25.1 & 31 & 26.8 \\
\hline
\end{tabular}

142 deviation; LOS: length of stay; ICU: intensive care unit

143 We found no statistical difference regarding the day of arrival for patients in all trigger teams. Looking at

144 time of arrival, most trigger team patients arrive during daytime, with the amount of trauma patients

145 peaking in the early evening, STEMI patients around noon, and MEP in the morning. Hardly any patients

146 suspected for stroke arrive in the early morning hours.

147 Figure 2 shows the Kaplan-Meier survival graph for patients of the different trigger calls, with the

148 highest mortality for MEP.

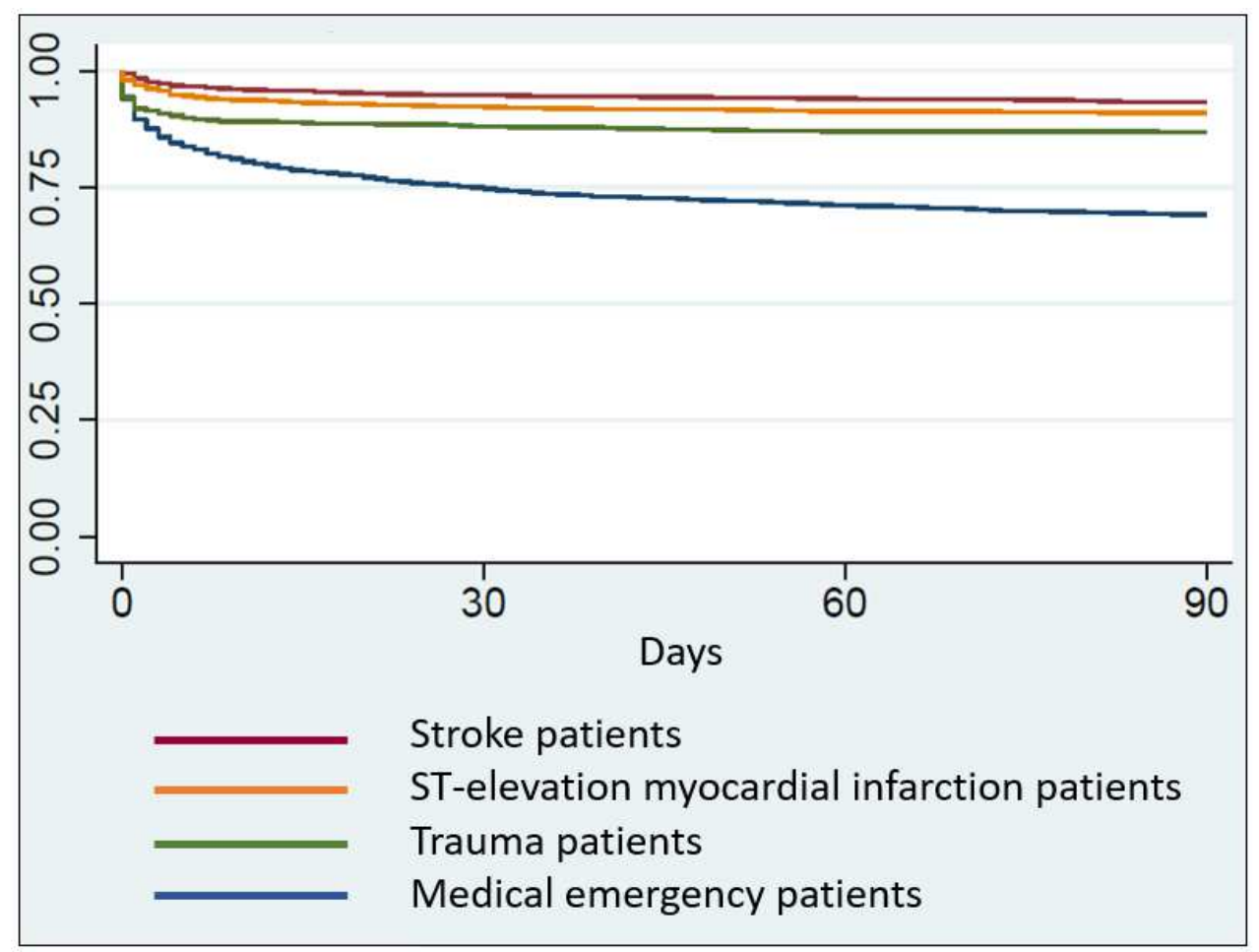


Figure 2: Kaplan-Meier survival estimates

151

152 The final diagnosis of patients included in the study are shown in figure 3 . Whereas patients from

153 trauma, STEMI and stroke calls were mainly discharged within a few ICD10 main coding areas, patients

154 from MEP calls had discharge diagnosis within many different ICD10 main coding areas.

155

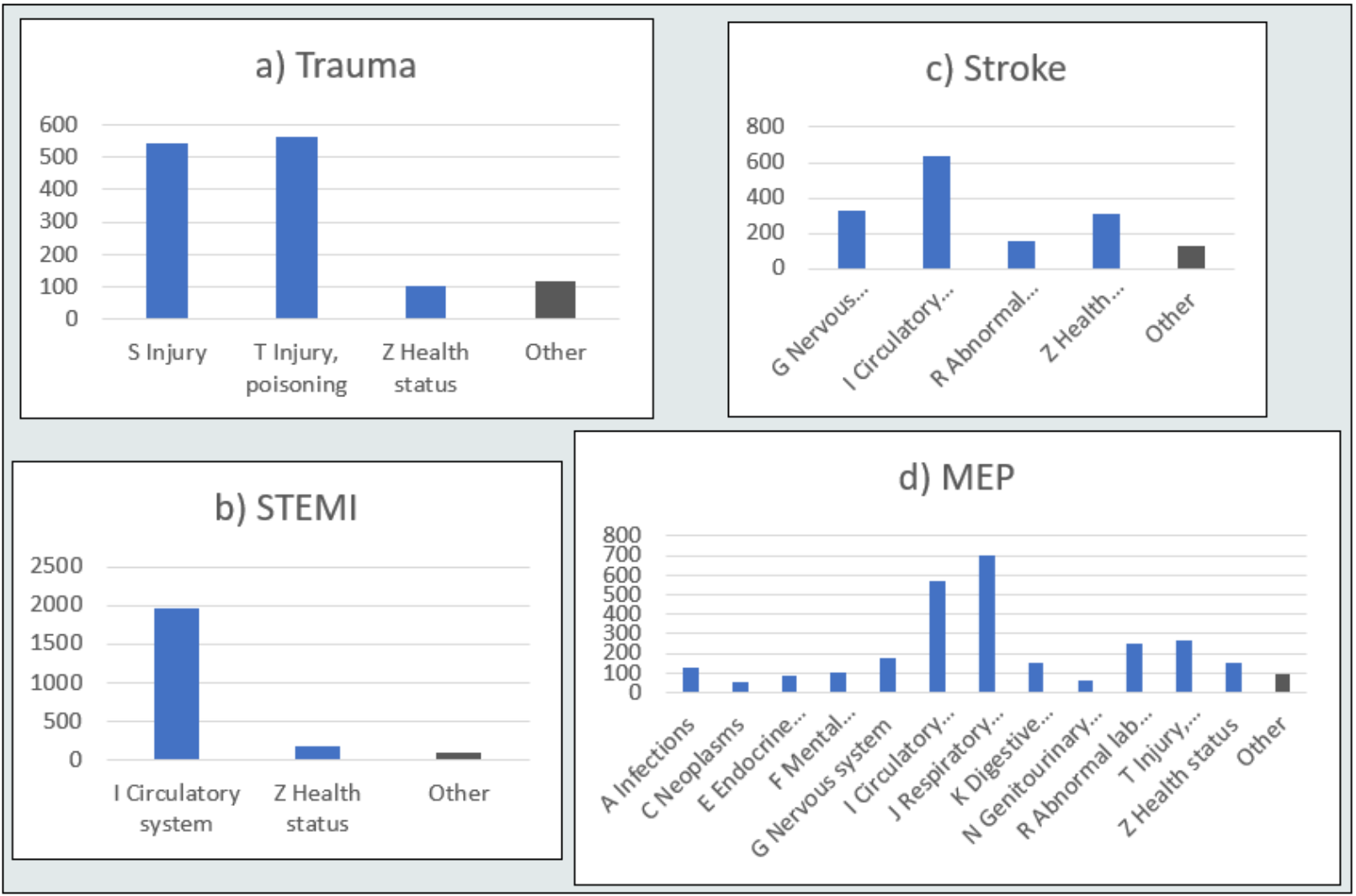

157 Figure 3: Final diagnosis (International classification of diseases, version 10)

158 a) Trauma patients, b) STEMI patients, c) Stroke patients, d) MEP

159 STEMI: ST-elevation myocardial infarction; MEP: medical emergency patients 


\section{Discussion:}

164 Huge improvements regarding their outcome were made in recent years for patients with trauma, 165 stroke and STEMI. There is a lack of national databases and guidelines for MEP to reach comparable 166 successes for this patient group. MEP are complex, have a low chance of survival, and they are frequent.

167 There are well established databases for patients with trauma (Danish Trauma Registry, TraumaRegister 168 DGU), STEMI (Acute Coronary Syndrome STEMI (ACS) Registry) and stroke (SITS - Safe Implementation 169 of Treatment in Stroke), with published studies illuminating their mortality and outcomes (13-16).

170 Similar databases for MEP are lacking, and only few studies have been published describing this patient 171 group $(4,5,7,17-21)$. The survival rates and base characteristics for patients with trauma, STEMI and 172 suspected stroke at OUH match those published in international databases $(13,14,16)$.

173 In this study, we were able to compare the outcome of all patients admitted by trigger teams to a level 1 174 university hospital. There are only 21 emergency hospitals in Denmark. OUH is the only hospital in a 175 perimeter of $50 \mathrm{~km}$, as well as the only level 1 hospital in the whole region. All patients fulfilling the 176 inclusion criteria for the different trigger calls were admitted here and could not be transported to an 177 alternate hospital. This makes comparison of the different patient groups possible and is one of the 178 strengths of the study.

179 In concordance with earlier studies $(18,21)$ we can show a high mortality rate for MEP, higher than for 180 the patients received by the other trigger teams. This is also true when comparing patients with 181 suspected stroke and MEP, who show approximately the same base statistics (i.e. age and gender). From 182 our data, we cannot conclude the reason for this, but it probably partially reflects the improved care 
183 after the introduction of thrombolysis, and a therefore reduced mortality rate for stroke patients. It

184 could also be a sign for a higher multimorbidity of MEP.

185 Looking at ICU admission, two groups of patients are sticking out, trauma patients and MEP. Both show 186 a high percentage of ICU admission (37.1\% and 26.8\%, respectively), but more MEP die within 90 days

187 after admission (13.2\% and 31\%, respectively).

188 Our results illustrate that we receive more MEP per 100,000 inhabitants than patients for the other 189 trigger teams. In other studies, it was not possible to show this divergence, as there are usually multiple 190 hospitals in the catchment areas, and there could be a distribution of the patients to different hospitals 191 according to the suspected severity of their acute symptoms.

192 It is not surprising that the final discharge diagnosis for patients with trauma, suspected stroke and 193 STEMI were limited to a narrow spectrum of the ICD10 catalogue. However, that is different for MEP. 194 Here, we found a wide distribution of diagnoses, ranging through the entire ICD10 catalogue. The most 195 common diagnosis groups were those describing respiratory and circulatory diseases. This shows the 196 inhomogeneity of this patient group and illuminates the complexity of developing diagnostic and 197 treatment guidelines for MEP that incorporate all possible aspects.

198 In 2014, a national register and a definition of MEP was requested in Germany (21), to better define the 199 patient population and develop guidelines. In Germany, in 2016, a consensus document was published, 200 with focus on patients arriving at the hospital with sepsis, amongst other diseases (23). Other MEP were 201 not included in the study. An Australian study could show that MEP received by an ICU based trigger 202 team had a shorter length of stay (24). Here, the setup did not include emergency physicians.

203 A study published in 2018 reviewed the outcome of MEP admitted in a similar set up as ours, with 204 equally high mortality, but also a much higher ICU admission (25). This probably reflects different local 
205 setups and lack of international guidelines. In Denmark, only one study is published focusing on MEP

206 (18).

207 MEP are heterogenic, and as there is no international agreement on their receival and treatment in the

208 ED, their path through the ED differs from hospital to hospital. A review from 2017 concluded that it was

209 not possible to suggest practice recommendations for MEP (22). Reason for this was the lack of

210 sufficient data on MEP.

211

212 Our study has limitations. Some patients with cardiac arrest could have been coded as STEMI patients or

213 MEP, thus raising the 7-day mortality. The study was composed as a single centre study and reflects the

214 local setup for the trigger teams. We have no information on base values, Charlson comorbidity index,

215 or lab findings to compare the different patient groups.

216

217

218

219 Conclusion:

220 In this study, the amount and outcome of patients received by specialized teams for trauma, stroke,

221 STEMI and MEP at a level 1 hospital can be compared, as there is definiteness regarding the catchment

222 areas. MEP have a higher mortality and ICU admission rate than patients of the other groups. Their final

223 diagnosis ranges through the whole ICD10 catalogue.

224 
227 List of abbreviations:

228 STEMI ST-elevation myocardial infarction

229 MEP Medical emergency patients

230 ED Emergency department

231 OUH Odense University Hospital

232 LPR National Patient Registry (Landspatientregisteret)

233 SD Standard deviation

234 LOS Length of stay

235 ICU Intensive care unit

236 ICD10 International classification of diseases, version 10

237 SITS Implementation of Treatments in Stroke

238

239

240

241 Declarations:

242 Ethics approval and consent to participate: 
243 The study was approved by the Danish Health and Medicines Authority (File no. 3-3013-1385/1) and the

244 Danish Data Protection Agency (File no. 2013-41-2435). In accordance with Danish law no further

245 approval was needed. The reporting of this study conforms to the STROBE statement (26).

246 Consent for publication not applicable.

247

248 Availability of data and materials:

249 The datasets used and/or analysed during the current study are available from the corresponding author

250 on reasonable request.

251

252 Competing interests:

253 The authors declare that they have no competing interests.

254

255 Funding:

256 Annmarie Lassen is funded by an unrestricted grant from the philanthropic fund TrygFoundation given

257 to the University of Southern Denmark.

258

259 Authors' contributions: 

interpretation.

Acknowledgements:

Not applicable.

\section{References:}

1. Weile J, Nielsen K, Primdahl SC, Frederiksen CA, Laursen CB, Sloth E, et al. Trauma facilities in Denmark - A nationwide cross-sectional benchmark study of facilities and trauma care organisation. Scand J Trauma Resusc Emerg Med. 2018;26(1).

2. Groenewoudt M, Roest A. Septic patients arriving with emergency medical services: a seriously ill population. Eur J Emerg Med. 2013 [cited 2013 Dec 31]; Available from: http://europepmc.org/abstract/MED/24185258

3. Jones a E, Yiannibas V, Johnson C, Kline J a. Emergency department hypotension predicts sudden unexpected in-hospital mortality: a prospective cohort study. Chest. 2006 Oct [cited 2013 Dec 31];130(0012-3692 (Print) LA-eng PT-Journal Article SB-AIM SB-IM):941-6. Available from: http://www.ncbi.nlm.nih.gov/pubmed/17035422

4. Boniatti MM, Azzolini N, Viana M V, Ribeiro BSP, Coelho RS, Castilho RK, et al. Delayed Medical Emergency Team Calls and Associated Outcomes. Crit Care Med. 2013 Jan [cited 2013 Dec 24];42(c):1-5. Available from: http://www.ncbi.nlm.nih.gov/pubmed/23989173

5. Buist MD, Moore GE, Bernard SA, Waxman BP, Anderson JN, Nguyen T V. Effects of a medical emergency team on reduction of incidence of and mortality from unexpected cardiac arrests in hospital: preliminary study. BMJ. 2002;324(7334):387-90.

6. Goldhill DR, Worthington L, Mulcahy a, Tarling M, Sumner a. The patient-at-risk team: identifying and managing seriously ill ward patients. Anaesthesia. 1999;54(9):853-60.

7. Considine J, Jones D, Bellomo R. Emergency department rapid response systems: the case for a standardized approach to deteriorating patients. Eur J Emerg Med. 2013 Dec [cited 2013 Dec 31];20(6):375-81. Available from: http://www.ncbi.nlm.nih.gov/pubmed/23325146 
8. Ranji SR, Auerbach AD, Hurd CJ, O'Rourke K, Shojania KG. Effects of rapid response systems on clinical outcomes: systematic review and meta-analysis. J Hosp Med. 2007 Dec [cited 2013 Dec 31];2(6):422-32. Available from: http://www.ncbi.nlm.nih.gov/pubmed/18081187

9. Salhi R, Edwards J, Gaieski D, Band R, Abella B, Carr B. Access to Care for Patients With TimeSensitive Conditions in Pennsylvania. Ann Emerg Med. 2013 Dec 21 [cited 2014 Feb 12]; Available from: http://www.ncbi.nlm.nih.gov/pubmed/24368055

10. Schmidt M, Pedersen L, Sørensen HT. The Danish Civil Registration System as a tool in epidemiology. Eur J Epidemiol. 2014 Aug [cited 2014 Nov 26];29(8):541-9. Available from: http://www.ncbi.nlm.nih.gov/pubmed/24965263

11. Schmidt M, Schmidt SAJ, Sandegaard JL, Ehrenstein V, Pedersen L, Sørensen HT. The Danish National patient registry: A review of content, data quality, and research potential. Vol. 7, Clinical Epidemiology. Dove Press; 2015 [cited 2019 Nov 22]. p. 449-90. Available from: https://www.dovepress.com/the-danish-national-patient-registry-a-review-of-content-dataquality--peer-reviewed-article-CLEP

12. Tiel Groenestege-Kreb D, Van Maarseveen O, Leenen L. Trauma team. Br J Anaesth. 2014;113(2):258-65. Available from: http://dx.doi.org/10.1093/bja/aeu236

13. Dyrvig A, Nielsen H, Larsen M. Dansk Traumeregister Årsrapport 2018. 2019;1-42

14. Hoefer C, Lefering R. TraumaRegister DGU Annual report 2019. 2019

15. TraumaRegister DGU. 20 years TraumaRegister DGU ${ }^{\circledR}$ : Development, aims and structure. Injury. 2014;45:S6-13. Available from: http://dx.doi.org/10.1016/j.injury.2014.08.011

16. Wahlgren N, Ahmed N, Caso V, Ford G, Lees K. Safe Implementation of Treatment in Stroke SITS Report 2018. 2018

17. Silva R, Saraiva M, Lage MJ, Campello G, Aragao I. The Medical Emergency Team in a universityaffiliated hospital: The past, the present and the future. Resuscitation. 2014 May [cited 2014 Sep 11];85:S103. Available from: http://linkinghub.elsevier.com/retrieve/pii/S0300957214003980

18. Jensen SM, Do HQ, Rasmussen SW, Rasmussen LS, Schmidt TA. Emergency team calls for critically ill non-trauma patients in the emergency department: An observational study. Scand J Trauma Resusc Emerg Med. 2015;23(1):2-7. Available from: http://dx.doi.org/10.1186/s13049-015-01592

19. Kumle B, Merz S, Mittmann A, Pin M, Brokmann JC, Gröning I, et al. Non-traumatic resuscitation room management: Structure, organization, and initial measures. Notfall und Rettungsmedizin. 2019;402-14.

20. Bernhard M, Becker TK, Nowe T, Mohorovicic M, Sikinger M, Brenner T, et al. Introduction of a treatment algorithm can improve the early management of emergency patients in the resuscitation room. Resuscitation. 2007;73(3):362-73.

21. Bernhard, M., Ramshorn-Zimmer, A., Hartwig, T., Mende, L., Helm, M., Pega, J., \& Gries A. Management of critically ill patients in the resuscitation room. Different than for trauma? Vol. 63, Anaesthesist. 2014. p. 144-53. 
22. McDowald K, Direktor S, Hynes EA, Sahadeo A, Rogers ME. Effectiveness of collaboration between emergency department and intensive care unit teams on mortality rates of patients presenting with critical illness: a systematic review. Vol. 15, JBI database of systematic reviews and implementation reports. 2017. p. 2365-89.

23. Fischer M, Kehrberger E, Marung H, Moecke H, Prückner S, Trentzsch H, et al. The 2016 consensus document on emergency care for the general public during the pre-hospital phase and at the hospital. Notfall und Rettungsmedizin. 2016;19(5):387-95.

24. Durie ML, Darvall JN, Hadley DA, Tacey MA. A "Code ICU" expedited review of critically ill patients is associated with reduced emergency department length of stay and duration of mechanical ventilation. J Crit Care. 2017;42(2017):123-8. Available from: https://doi.org/10.1016/j.jcrc.2017.07.011

25. Bernhard M, Döll S, Hartwig T, Ramshorn-Zimmer A, Yahiaoui-Doktor M, Weidhase L, et al. Resuscitation room management of critically ill nontraumatic patients in a German emergency department (OBSERvE-study). Eur J Emerg Med. 2018;25(4):e9-17.

26. von Elm E, Altman DG, Pocock SJ, Gøtzsche PC, Vandenbroucke JP. Strengthening the reporting of observational studies in epidemiology (STROBE) statement: guidelines for reporting observational studies. BMJ. 2007 Oct;335(october):806-8. 
Figures

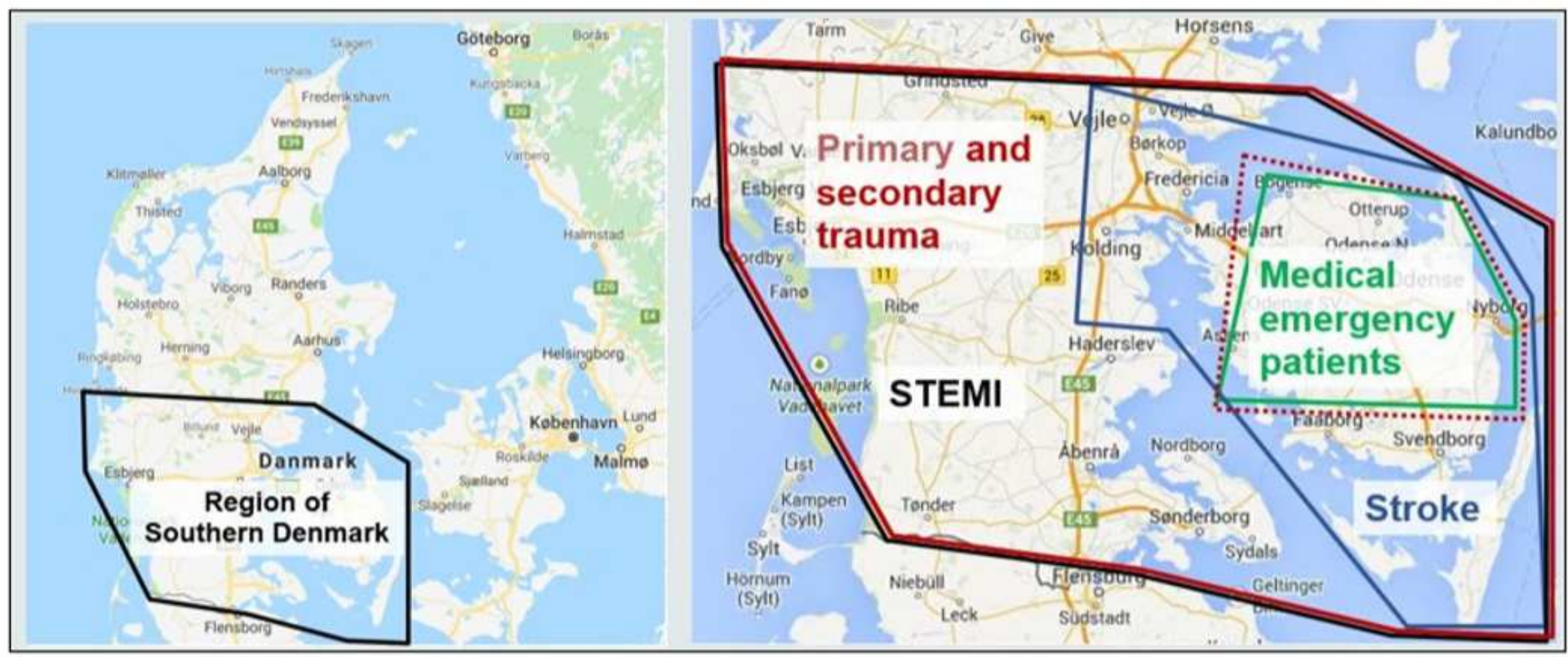

Figure 1

Catchment area STEMI: ST-elevation myocardial infarction patients 


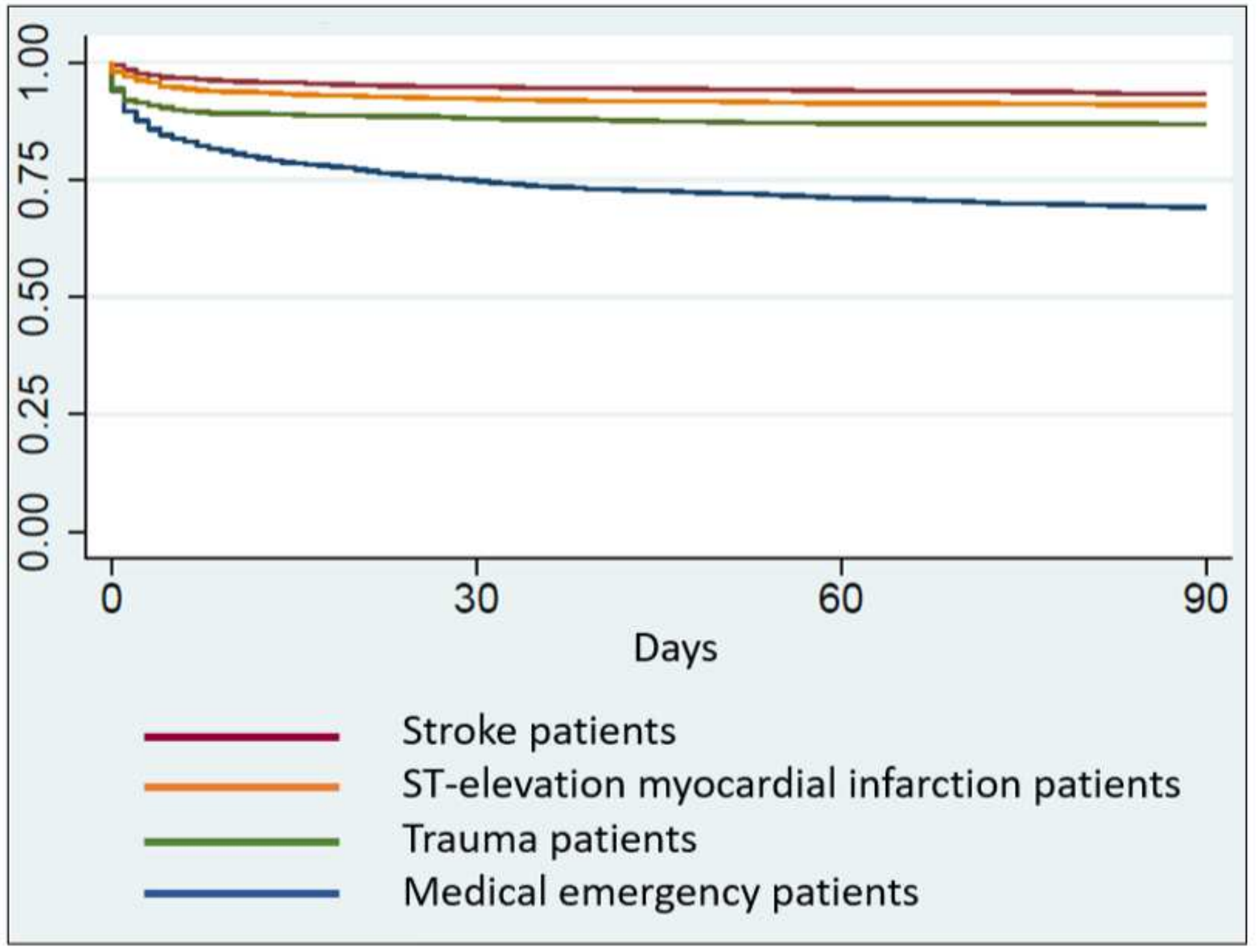

Figure 2

Kaplan-Meier survival estimates 


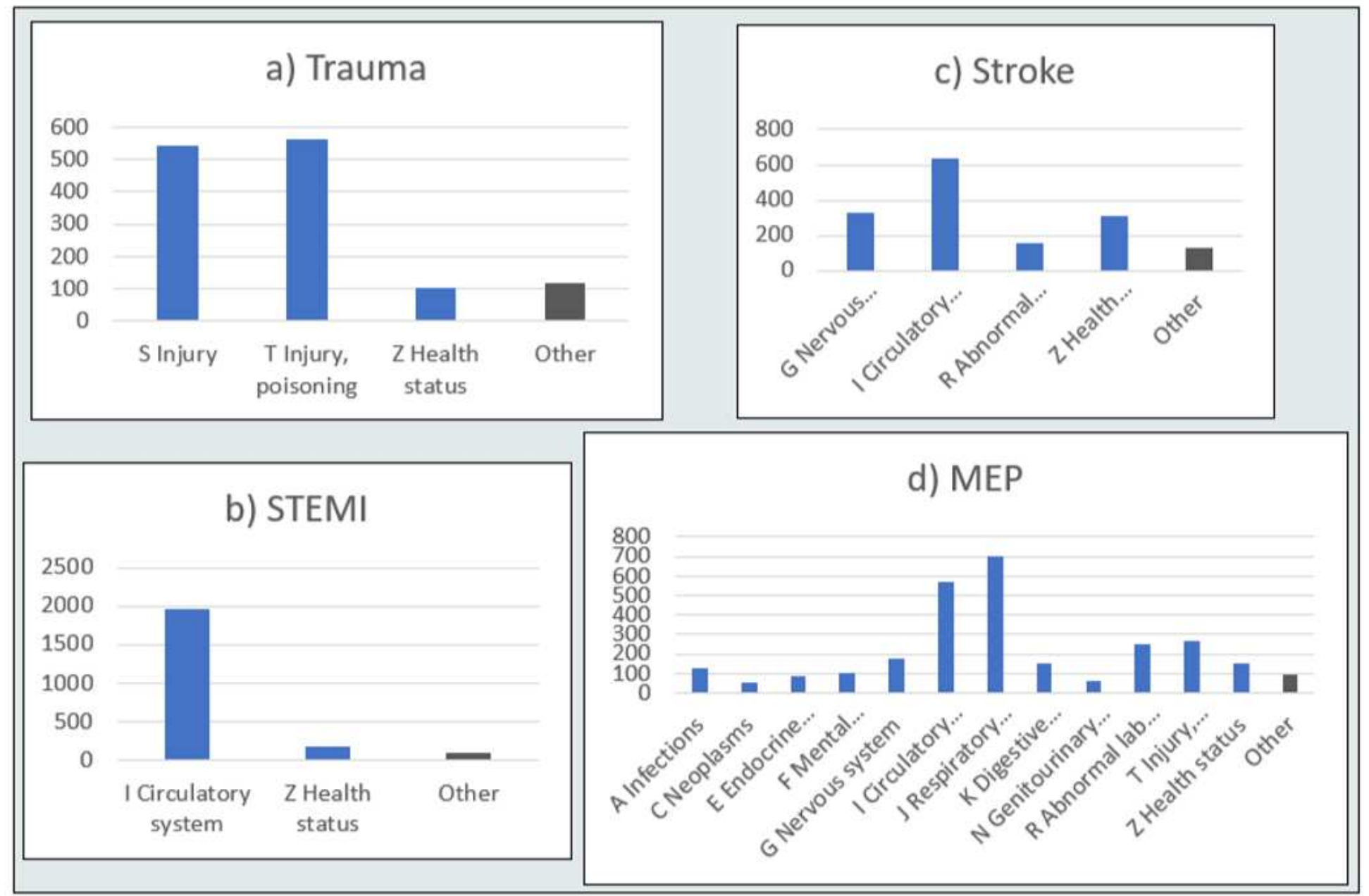

\section{Figure 3}

Final diagnosis (International classification of diseases, version 10) a) Trauma patients, b) STEMI patients, c) Stroke patients, d) MEP STEMI: ST-elevation myocardial infarction; MEP: medical emergency patients 